\title{
Radiomics MRI Phenotyping with Machine Learning to Predict the Grade of Lower-Grade Gliomas: A Study Focused on Nonenhancing Tumors
}

\author{
Yae Won Park, $M D^{1,2}$, Yoon Seong Choi, MD, PhD², Sung Soo Ahn, MD, PhD², Jong Hee Chang, MD, PhD², \\ Se Hoon Kim, MD, $\mathrm{PhD}^{4}$, Seung-Koo Lee, MD, $\mathrm{PhD}^{2}$ \\ ${ }^{1}$ Department of Radiology, Ewha Womans University College of Medicine, Seoul, Korea; ${ }^{2}$ Department of Radiology and Research Institute of \\ Radiological Science, Yonsei University College of Medicine, Seoul, Korea; Departments of ${ }^{3}$ Neurosurgery and ${ }^{4}$ Pathology, Yonsei University College \\ of Medicine, Seoul, Korea
}

Objective: To assess whether radiomics features derived from multiparametric MRI can predict the tumor grade of lower-grade gliomas (LGGs; World Health Organization grade II and grade III) and the nonenhancing LGG subgroup.

Materials and Methods: Two-hundred four patients with LGGs from our institutional cohort were allocated to training $(n=$ $136)$ and test $(n=68)$ sets. Postcontrast T1-weighted images, T2-weighted images, and fluid-attenuated inversion recovery images were analyzed to extract 250 radiomics features. Various machine learning classifiers were trained using the radiomics features to predict the glioma grade. The trained classifiers were internally validated on the institutional test set and externally validated on a separate cohort $(n=99)$ from The Cancer Genome Atlas (TCGA). Classifier performance was assessed by determining the area under the curve (AUC) from receiver operating characteristic curve analysis. An identical process was performed in the nonenhancing LGG subgroup (institutional training set, $n=73$; institutional test set, $n=37$; and TCGA cohort, $\mathrm{n}=37$ ) to predict the glioma grade.

Results: The performance of the best classifier was good in the internal validation set (AUC, 0.85) and fair in the external validation set (AUC, 0.72) to predict the LGG grade. For the nonenhancing LGG subgroup, the performance of the best classifier was good in the internal validation set (AUC, 0.82), but poor in the external validation set (AUC, 0.68).

Conclusion: Radiomics feature-based classifiers may be useful to predict LGG grades. However, radiomics classifiers may have a limited value when applied to the nonenhancing LGG subgroup in a TCGA cohort.

Keywords: Grade; Lower-grade glioma; Magnetic resonance imaging; Radiomics; The Cancer Genome Atlas

\section{INTRODUCTION}

Diffuse lower-grade gliomas (LGGs), which are analogous to World Health Organization (WHO) grade II and grade III gliomas (1), are infiltrative neoplasms that most often arise in the cerebral hemispheres of adults. Although the 2016 update to the WHO classification reflects a combination of phenotypic and genotypic information (2), the therapeutic strategy is still heavily reliant on the WHO grade and patients' prognosis differs significantly according to tumor grade $(3,4)$. Although some recent studies have suggested that the prognostic differences between grade II and III are not as marked $(5,6)$, this has not been observed in all studies and grading is still important (7).

Biopsy results are the gold standard for glioma grading. However, the accuracy of biopsy may be limited by tumor

Received November 26, 2018; accepted after revision April 21, 2019.

This study was supported by a faculty research grant of Yonsei University College of Medicine (6-2016-0121).

Corresponding author: Yoon Seong Choi, MD, PhD, Department of Radiology and Research Institute of Radiological Science, Yonsei University College of Medicine, 50-1 Yonsei-ro, Seodaemun-gu, Seoul 03722, Korea.

- Tel: (822) 2228-7400•Fax: (822) 393-3035•E-mail: yoonseong.choi07@gmail.com

This is an Open Access article distributed under the terms of the Creative Commons Attribution Non-Commercial License (https:// creativecommons.org/licenses/by-nc/4.0) which permits unrestricted non-commercial use, distribution, and reproduction in any medium, provided the original work is properly cited. 
heterogeneity and sampling errors, which may result in tumor undergrading (8-10). Moreover, biopsy itself is an invasive technique with reported morbidity and mortality rates of up to $3.6 \%$ and $1.7 \%$, respectively (11, 12). Therefore, if grade III LGGs are suspected, a more aggressive surgical resection should be considered to avoid unnecessary biopsy (13). In preoperative grading of LGGs, the presence and degree of contrast enhancement have been traditionally used as a hallmark of grade III gliomas (14-16). However, several studies have demonstrated that the absence of enhancement does not necessarily imply WHO grade II glioma (17-19), and grade III gliomas have been reported to comprise $34.3-42.3 \%$ of nonenhancing LGGs $(13,18-21)$, making preoperative grading of nonenhancing $L G G$ s difficult.

Radiomics features show tumor characteristics noninvasively by extracting the high-dimensional quantitative features that reflect tumor morphology and heterogeneity (22). Several studies have reported that radiomics features can be used to predict the genetic subtypes and prognosis of gliomas $(23,24)$. However, the role of these features in the prediction of LGG grade has not yet been established. We hypothesized that radiomics features can extract invisible information regarding glioma grade.

The aim of this study was to assess whether radiomics features from multiparametric MRI can predict tumor grade in LGGs by using machine learning. The assessments also focused on the nonenhancing LGG subgroup, which is often classified as WHO grade II glioma on preoperative MRI.

\section{MATERIALS AND METHODS}

\section{Patient Population}

The Institutional Review Board waived the need for obtaining informed patient consent for this retrospective study. Between September 2007 and January 2017, 231 patients with pathologically diagnosed WHO grade II and III gliomas from our institution were included in this study. Inclusion criteria were as follows: 1 ) WHO grade II or III gliomas confirmed by histopathology, 2) patients who underwent preoperative MRI, and 3) patients aged $\geq 18$ years. The exclusion criteria were as follows: 1 ) absence of T2-weighted or fluid-attenuated inversion recovery (FLAIR) images $(n=19) ; 2)$ a previous history of biopsy or operation for brain tumors $(n=3)$; and 3 ) errors in image processing $(n=5)$. The mean interval between the MRI examination and operation was $13.4 \pm 14.1$ days. A total of 204 patients were finally enrolled in the institutional cohort. The institutional cohort was semirandomly allocated to training $(n=136)$ and test $(n=68)$ sets, with stratification for glioma grade.

The external validation set was collected from the LGG dataset from The Cancer Genome Atlas (TCGA), which is publicly available in The Cancer Imaging Archive (TCIA). For the 199 patients with publicly available magnetic resonance (MR) images from the TCGA cohort, the exclusion criteria were as follows: 1) absence of T2-weighted, FLAIR, or contrast-enhanced T1-weighted (T1C) images $(n=80) ; 2)$ previous history of biopsy or resection of tumor $(n=17)$; 3) severe motion artifact $(n=1)$; and 4) errors in image processing $(n=2)$. Finally, 99 patients were enrolled in the TCGA cohort.

For nonenhancing LGG subgroup analysis, 110 of the 204 patients from the institutional cohort were enrolled and semirandomly allocated to training $(n=73)$ and test $(n=$ 37) sets, with stratification for glioma grade. Similarly, 37 out of the 99 patients from the TCGA cohort were enrolled in the nonenhancing LGG subgroup and used for external validation. The patient enrollment process for the entire LGG group and the nonenhancing LGG subgroup is shown in Figure 1.

\section{Pathological Diagnosis}

For pathological diagnosis of glioma grade in the institutional cohort, surgical specimens were fixed in $10 \%$ buffered formaldehyde in preparation for light microscopy and stained with hematoxylin-eosin. Histologic grading was performed according to the WHO classification by a neuropathologist (16 years of experience) who was blinded to the clinical information (2). For the TCGA cohort, the histological characteristics and grading of tissues submitted to TCGA were confirmed by a neuropathology review, as previously described $(1,25)$.

\section{MRI Protocol}

Preoperative MRI examinations for the institutional cohort were performed using a 3T MRI scanner (Achieva, Philips Healthcare, Best, The Netherlands) with an eightchannel sensitivity-encoding head coil. The preoperative MRI protocol included T1-weighted (repetition time [TR]/ echo time [TE], 1800-2000/10-15 ms; field of view [FOV], $240 \mathrm{~mm}$; section thickness, $5 \mathrm{~mm}$; matrix, $256 \times 256$ ), T2weighted (TR/TE, 2800-3000/80-100 ms; FOV, 240 mm; 


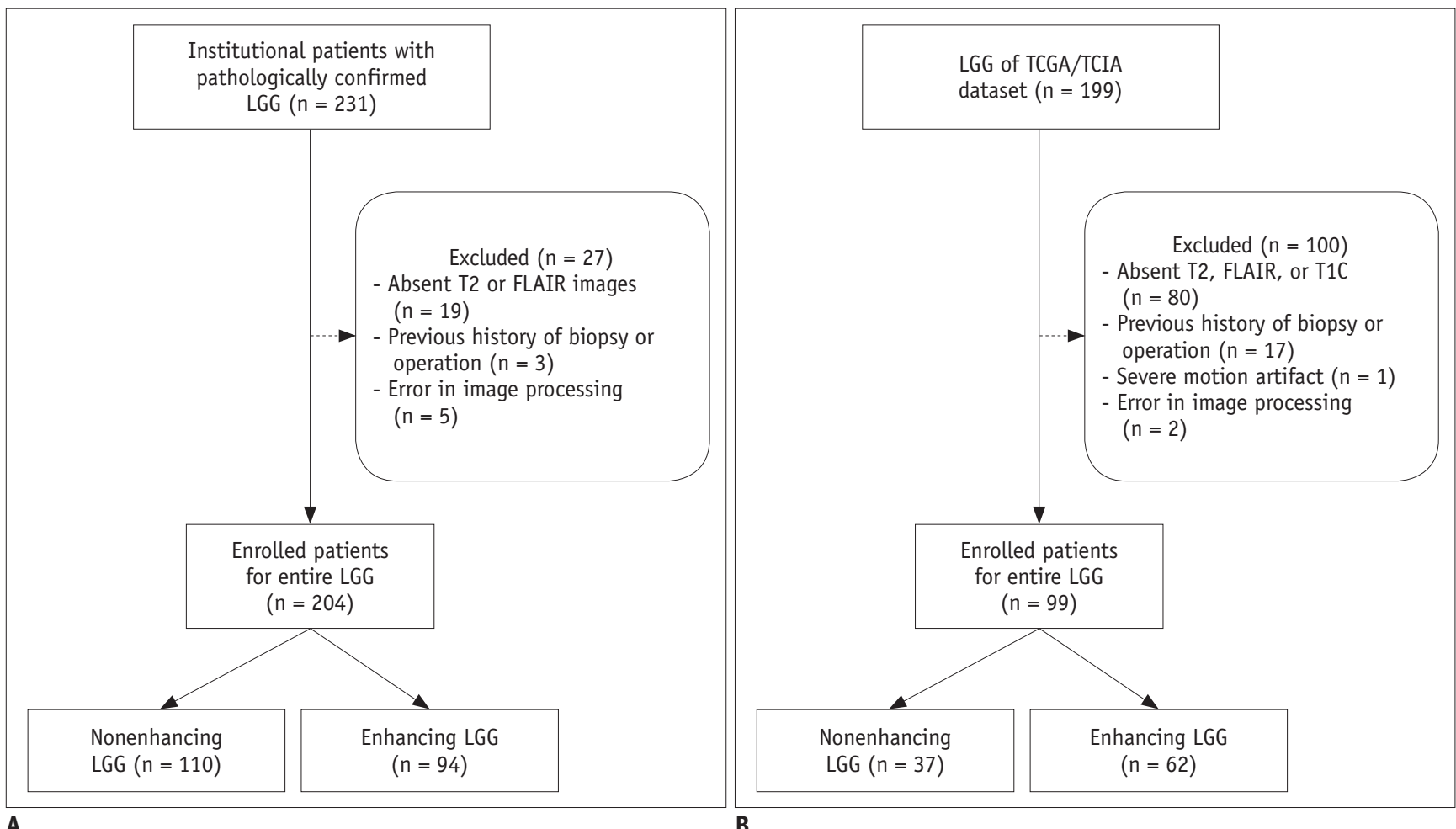

Fig. 1. Patient enrollment process for entire LGG group and nonenhancing LGG subgroup in (A) institutional cohort and (B) TCGA cohort. FLAIR = fluid-attenuated inversion recovery, LGG = lower-grade glioma, TCGA $=$ The Cancer Genome Atlas, TCIA = The Cancer Imaging Archive, T1C = contrast-enhanced T1-weighted

section thickness, $5 \mathrm{~mm}$; matrix, $256 \times 256)$, and FLAIR (TR/TE, 9000-10000/110-125 ms; FOV, 240 mm; section thickness, $5 \mathrm{~mm}$; matrix, $256 \times 256$ ) sequences. Threedimensional T1C images (TR/TE, 6.3-8.3/3.1-4 ms; FOV, $240 \mathrm{~mm}$; section thickness, $1 \mathrm{~mm}$; and matrix, $192 \times 192$ ) were acquired after administering $0.1 \mathrm{~mL} / \mathrm{kg}$ of gadoliniumbased contrast material (gadobutrol [Gadovist], Bayer AG, Toronto, Canada).

The different MRI scanners for the TCGA cohort acquired from five institutions is listed on Supplementary Table 1. Further detailed information on the diverse imaging protocols for this study in available online (https://www. nature.com/articles/sdata2017117/tables/3) (26).

\section{Image Processing and Radiomics Feature Extraction}

To overcome the heterogeneity in the different MRI protocols, the T2-weighted images from the institutional and TCGA/TCIA cohorts were resampled to $0.5 \times 0.5 \mathrm{~mm}$ spatial resolution and 5-mm slice thickness and used as a registration template for FLAIR and T1C images. After skull stripping, the T1C and FLAIR images were registered to the T2-weighted image template by using FMRIB software library (FSL, http://fsl.fmrib.ox.ac.uk/fsl/fslwiki/FSL). Image signal intensities were normalized using the WhiteStripe $\mathrm{R}$ package (27). Region-of-interests (ROIs) covering the entire tumor including edema (defined as hyperintense areas on FLAIR), were drawn semiautomatically by using thresholds of signal intensity and by consensus of two neuroradiologists (with 10 years and 7 years of experience, respectively). In total, 250 radiomics features were extracted from the ROIs on T1C, T2-weighted, and FLAIR images, which were related to shape $(n=16)$, first-order statistics $(n=19)$, gray level co-occurrence matrix $(n=27)$, gray level runlength matrix $(n=16)$, and gray level size zone matrix ( $n$ = 16) (Supplementary Table 2). The feature extraction was performed with Pyradiomics 1.2.0 (http://www.radiomics. io/pyradiomics.html) (28).

\section{Statistical Analysis and Machine Learning}

The workflow for machine learning after image processing and feature extraction is shown in Figure 2. Various machine learning classifiers were trained to predict the glioma grade in the entire LGG group and the nonenhancing subgroup. For feature selection, Student's $t$ test according to glioma grade was performed for each radiomics feature to filter the features with $p<0.2$. In 


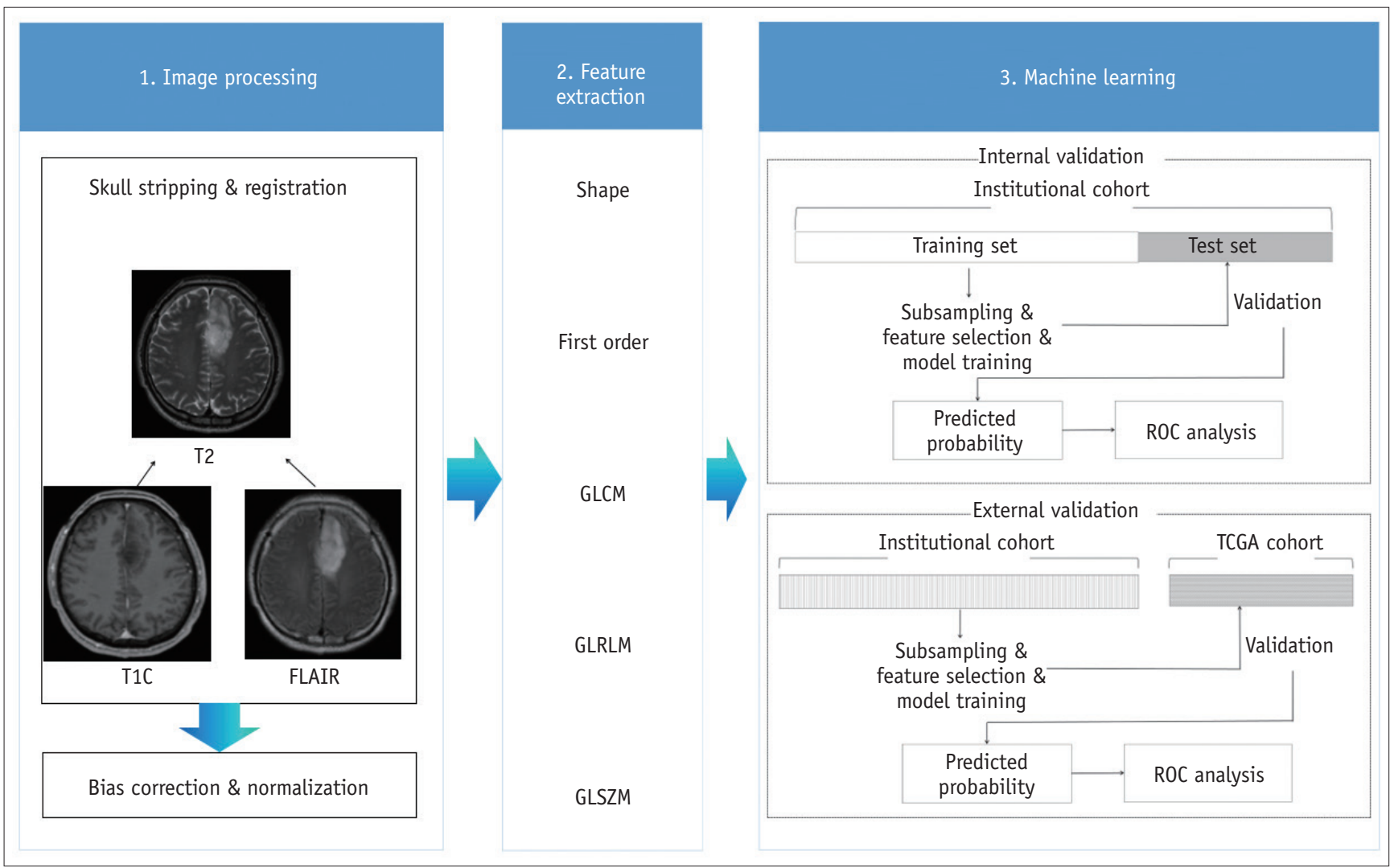

Fig. 2. Workflow of image processing, radiomics feature extraction, and machine learning. GLCM = gray level co-occurrence matrix, GLRLM = gray level run-length matrix, GLSZM = gray level size zone matrix, ROC = receiver operating characteristic

some classifiers, recursive feature elimination (RFE) was additionally performed for those features filtered from the $t$ test. These feature selection methods were combined with the elastic net, random forest (RF), gradient boosting machine (GBM), and linear discriminant analysis (LDA) algorithms, resulting in five combinations: 1) $t$ test, RFE, and $\mathrm{RF}, 2) t$ test, RFE, and GBM, 3$) t$ test, RFE, and LDA, 4) $t$ test and elastic net, and 5) $t$ test and RF. Therefore, five radiomics classifiers were trained to classify tumor grade in the entire LGG group. In the nonenhancing LGG subgroup, to overcome data imbalance, subsampling with the random over-sampling examples (ROSE) and synthetic minority over-sampling technique (SMOTE) methods or no subsampling were additionally performed after univariate filtering by the $t$ test and combined with the previously mentioned five radiomics classifiers. Therefore, a total of 15 radiomics classifiers were trained for the nonenhancing $L G G$ subgroup (29). During classifier training, hyperparameters of classification algorithms were optimized by grid search.

The radiomics classifiers were validated internally on the institutional test set and externally on the TCGA cohort. In the entire LGG group, for internal validation, radiomics classifiers were trained on the institutional training set $(n=$ 136) and validated on the institutional test set $(n=68)$. For external validation, the classifiers were trained on the entire institutional cohort $(n=204)$ and validated on the TCGA cohort $(n=99)$. The radiomics classifier performance was measured by performing receiver operating characteristics analysis and calculating the area under the curve (AUC). The accuracy, sensitivity, specificity, and no-information rate (percentage of the majority class, i.e., the accuracy determined by assuming that all samples belong to majority class) were also calculated for the best classifiers with the highest AUC values. In addition, an one-sided hypothesis test was performed using the "binom.test" function to evaluate whether the radiomics classifier accuracy is significantly higher than the no-information rate.

An identical process of training and validation was performed for 15 machine learning classifiers in the nonenhancing LGG subgroup. For internal validation, machine learning classifiers were trained on the institutional training set $(n=73)$ and validated on the institutional test set $(n=$ 37). For external validation, the classifiers were trained on the entire nonenhancing institutional cohort $(n=110)$ and 
validated on the nonenhancing TCGA cohort $(n=37)$.

Statistical analysis was performed using the statistical software R (version 3.3.1; R Foundation for Statistical Computing, Vienna, Austria). The RFE feature selection and classification algorithms were implemented using the "caret" R package (30). ROSE and SMOTE subsampling was performed using the "ROSE" and "DMwR" R packages. Statistical significance was set at $p<0.05$.

\section{RESULTS}

The patient characteristics in the institutional training ( $\mathrm{n}$ $=136$ and 73 for the entire group and nonenhancing LGG subgroup, respectively) and test sets ( $n=68$ and 37 for the entire group and nonenhancing LGG subgroup, respectively) and the TCGA validation set $(n=99$ and 37 for the entire group and nonenhancing LGG subgroup, respectively) are summarized in Tables 1 and 2. The proportion of nonenhancing LGGs differed in the institutional and TCGA cohorts (76\% in the institutional cohort and $56.3 \%$ in the TCGA cohort for grade II gliomas and $22.4 \%$ in the institutional cohort and $19.6 \%$ in the TCGA cohort for grade
III gliomas).

The AUC values for predicting glioma grade by various machine learning classifiers are shown in Figure 3. Summaries of the performance of the best machine learning classifiers in the internal and external validation of the entire LGG group and nonenhancing LGG subgroup are presented in Table 3. In internal validation with the entire LGG group, the best performance was shown by a combination of feature selection by the $t$ test and elastic net without subsampling, with an AUC, accuracy, sensitivity, and specificity of $0.85,79.4 \%, 92.9 \%$, and $70.0 \%$, respectively. In external validation with the entire LGG subgroup, the best performance was achieved by a combination of feature selection using the $t$ test and RFE and the GBM algorithm, with an AUC, accuracy, sensitivity, and specificity of $0.72,66.7 \%, 72.6 \%$, and $60.4 \%$, respectively. The accuracy of the radiomics classifier was significantly higher than the no-information rate in both internal $(79.4 \%$ vs. $58.8 \%, p<0.001)$ and external $(66.7 \%$ vs. $51.5 \%, p=0.002)$ validations.

In internal validation with the nonenhancing $L G G$ subgroup, the best classifier was the combination of $t$ test

Table 1. Patient Characteristics in Entire LGG Group

\begin{tabular}{|c|c|c|c|c|c|}
\hline \multirow{2}{*}{ Variables } & \multicolumn{3}{|c|}{ Institutional Cohort $(n=204)$} & \multirow{2}{*}{ TCGA Validation Set $(n=99)$} & \multirow{2}{*}{$P^{*}$} \\
\hline & Training Set $(n=136)$ & Test Set $(n=68)$ & Total $(n=204)$ & & \\
\hline Age (mean \pm SD) & $44.99 \pm 12.94$ & $44.00 \pm 12.33$ & $44.66 \pm 12.74$ & $46.96 \pm 13.95$ & 0.154 \\
\hline Sex & & & & & 0.222 \\
\hline Male & $65(47.8)$ & $43(63.2)$ & $108(52.9)$ & $55(55.6)$ & \\
\hline Female & $71(52.2)$ & $25(36.8)$ & $96(47.1)$ & $54(44.4)$ & \\
\hline Grade & & & & & 0.075 \\
\hline II & $81(59.6)$ & $40(58.8)$ & $121(59.3)$ & $48(48.5)$ & \\
\hline III & $55(40.4)$ & $28(41.2)$ & $83(40.7)$ & $51(51.5)$ & \\
\hline
\end{tabular}

Data are number of patients. Numbers in parentheses are percentage. * $p$ values were calculated using Student's $t$ test for continuous variables and chi-square test for categorical variables, to compare patient characteristics of institutional cohort $(n=204)$ and TCGA validation set $(\mathrm{n}=99)$. LGG = lower-grade glioma, $\mathrm{SD}=$ standard deviation, TCGA = The Cancer Genome Atlas

Table 2. Patient Characteristics in Nonenhancing LGG Subgroup

\begin{tabular}{lcccc}
\hline \multirow{2}{*}{ Variables } & \multicolumn{3}{c}{ Institutional Cohort $(\mathrm{n}=110)$} & \multirow{2}{*}{ TCGA Validation Set $(\mathrm{n}=37)$} \\
\cline { 2 - 4 } $\begin{array}{l}\text { Age }(\text { mean } \pm \text { SD) } \\
\text { Sex }\end{array}$ & $43.18 \pm 11.76$ & $43.32 \pm 10.28$ & $44.24 \pm 11.34$ & $43.56 \pm 15.13$ \\
$\quad$ Male & $39(53.4)$ & $14(37.8)$ & $53(48.2)$ & $17(45.9)$ \\
$\quad$ Female & $34(46.6)$ & $23(62.2)$ & $57(51.8)$ & $20(54.1)$ \\
Grade & $61(83.6)$ & $31(83.8)$ & $92(83.6)$ & $27(73.0)$ \\
$\quad$ II & $12(16.4)$ & $6(16.2)$ & $18(16.4)$ & $10(27.0)$ \\
\hline III & & & & 0.247 \\
\hline
\end{tabular}

Data are number of patients. Numbers in parentheses are percentage. * $p$ values were calculated using Student's $t$ test for continuous variables and chi-square test for categorical variables, to compare patient characteristics of institutional cohort $(n=110)$ and TCGA validation set $(n=37)$. 


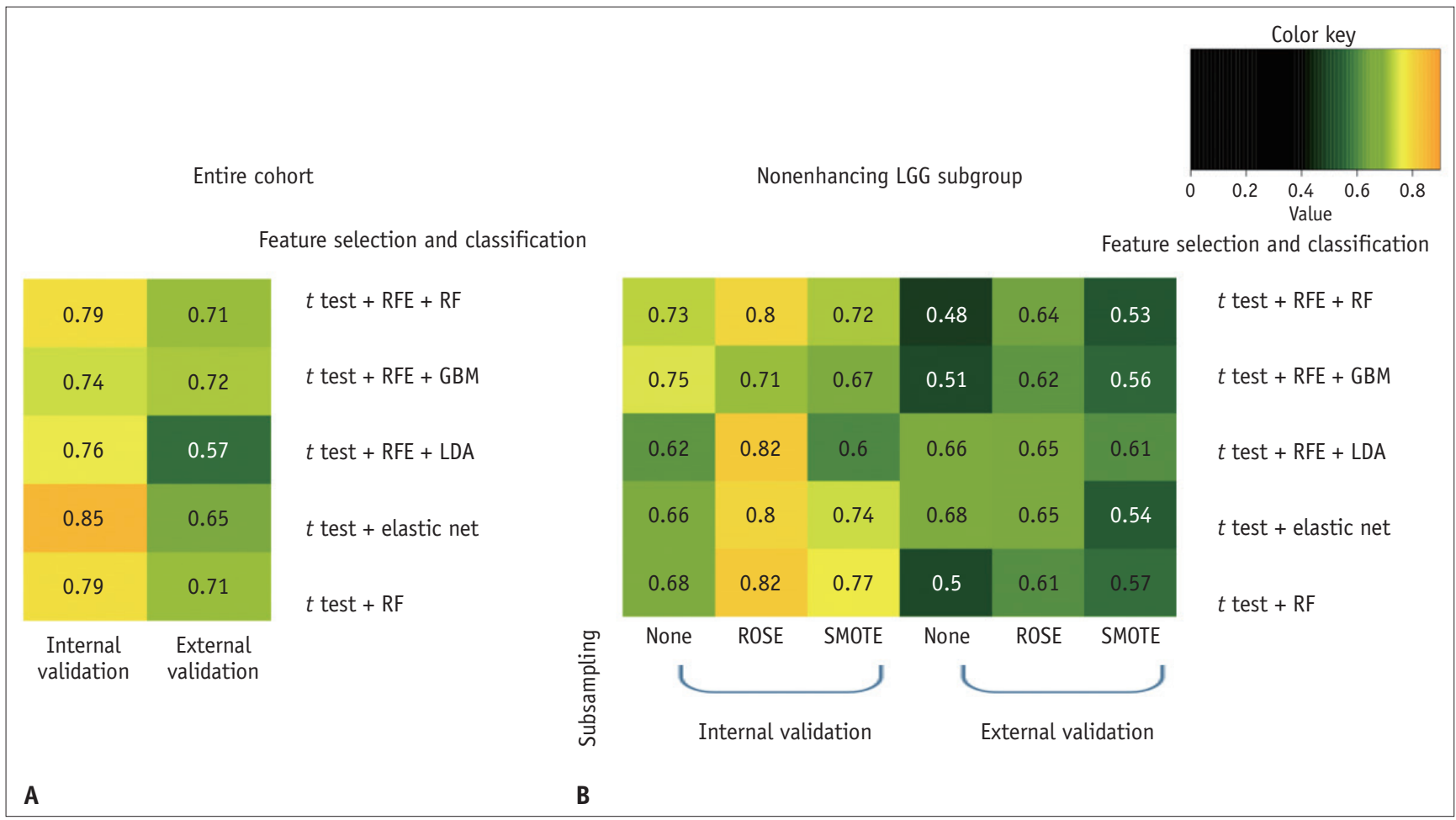

Fig. 3. Heatmap of AUC values. Heat map of AUC values from machine learning classifier to predict grade (A) in entire LGG group in internal validation for institutional test set $(n=136)$ after training on institutional training set $(n=68)$ and entire LGG group in external validation for TCGA validation set $(n=99)$ after training on entire institutional cohort $(n=204)$; and $(B)$ in nonenhancing LGG subgroup in internal validation for institutional test set $(n=73)$ after training on institutional training set $(n=37)$ and nonenhancing LGG subgroup in external validation on TCGA cohort $(n=37)$ after training on entire nonenhancing institutional cohort $(n=110)$. AUC $=$ area under curve, GBM $=$ gradient boosting machine, $\mathrm{LDA}=$ linear discriminant analysis, $\mathrm{RF}=$ random forest, $\mathrm{RFE}=$ recursive feature elimination, $\mathrm{ROSE}=$ random over-sampling examples, SMOTE = synthetic minority over-sampling technique

Table 3. Performance of Best Machine Learning Classifiers in Grade Prediction for Entire LGG Group and Nonenhancing LGG Subgroup in Internal and External Validations

\begin{tabular}{|c|c|c|c|c|c|c|c|c|}
\hline Cohort & Validation & Subsampling & AUC (95\% CI) & Accuracy & Sensitivity & Specificity & NIR & $P$ \\
\hline Entire LGG & Internal & $t$ test + elastic net & $0.85(0.76-0.94)$ & $79.4 \%$ & $92.9 \%$ & $70.0 \%$ & $58.8 \%$ & $<0.001$ \\
\hline Entire LGG & TCGA & $t$ test + RFE + GBM & $0.72(0.62-0.82)$ & $66.7 \%$ & $72.6 \%$ & $60.4 \%$ & $51.5 \%$ & 0.002 \\
\hline Nonenhancing LGG & Internal & $t$ test + ROSE + RF & $0.82(0.66-0.97)$ & $78.4 \%$ & $83.3 \%$ & $77.4 \%$ & $83.8 \%$ & 0.866 \\
\hline Nonenhancing LGG & TCGA & $t$ test + elastic net & $0.68(0.49-0.87)$ & $72.2 \%$ & $55.6 \%$ & $77.8 \%$ & $75.0 \%$ & 0.725 \\
\hline
\end{tabular}

$\mathrm{AUC}=$ area under curve, $\mathrm{CI}=$ confidence interval, $\mathrm{GBM}=$ gradient boosting machine, NIR $=$ no-information rate, $\mathrm{RF}=$ random forest, $\mathrm{RFE}=$ recursive feature elimination, $\mathrm{ROSE}=$ random over-sampling examples

feature selection, RF algorithm, and ROSE subsampling, with AUC, accuracy, sensitivity, and specificity of 0.82 , $78.4 \%, 83.3 \%$, and $77.4 \%$, respectively. In external validation with the nonenhancing LGG subgroup, however, all 15 machine learning classifiers failed to show good performance (AUC, $0.48-0.68$ ) regardless of subsampling. The best classifier was the combination of $t$ test feature selection and elastic net without subsampling, with an AUC, accuracy, sensitivity, and specificity of $0.68,72.2 \%, 55.6 \%$, and $77.8 \%$, respectively.

\section{DISCUSSION}

In this study, we aimed to differentiate the LGG grades using radiomics features from routinely obtained MRI sequences. Our results showed that radiomics classifiers are useful to predict the tumor grade of LGGs, although radiomics classifiers may have a limited value for grading the nonenhancing LGG subgroup, especially in a heterogeneous external cohort.

Previous studies have attempted to differentiate the grades of LGG by using various advanced imaging 
techniques, such as diffusion tensor imaging, perfusionweighted imaging, MR spectroscopy, and C-methionine positron emission tomography $(13,18-21,31,32)$. However, most studies recruited smaller sample sizes and included the entire LGG group regardless of enhancement. Only a few studies analyzed the nonenhancing LGG subgroup and reported a limited performance of the imaging biomarkers in grading nonenhancing $\operatorname{LGG}(13,18)$. Moreover, many of these studies used advanced imaging techniques that might not be routinely performed and therefore had limited feasibility. In our study, however, routine conventional MR sequences were analyzed using a pipeline that was built completely upon open-source packages. The use of common MR sequences and easily accessible tools may yield a more feasible methodology and reproducible results that can be shared with other institutions. High-throughput quantitative radiomics features are prone to yield information imperceptible to the human eye, reflecting the disruption or compression of normal anatomy by tumors, vasogenic edema, and tumor cellularity. Recent studies have reported that texture analysis is a potentially useful approach for estimating the molecular status in LGGs (33, 34), and we hypothesized that multiparametric MR features may be also useful in LGG grading.

In this study, we used the institutional training set and the TCGA test set. This allowed us indirectly to assess how the differences in MR protocols can affect the model performance and whether radiomics phenotyping can still provide useful information regarding the LGG grade across the different MR protocols. The accuracy of grading for the entire LGG group was significantly greater than the no-information rate in both internal and external validations. This result implies that radiomics is useful for LGG grading, despite the heterogeneity in the TCGA cohort with the different imaging protocols. Nonetheless, as the AUC in the external validation was lower than that in the internal validation for both the entire LGG group and the nonenhancing LGG subgroup, our results also imply that standardization of MRI protocols is mandatory to achieve optimized performance of radiomics classifiers. In addition, it should be emphasized that although the best radiomics classifier is useful for differentiating LGG grades, a diverse range of performance levels was noted according to the machine learning algorithms and resampling methods used. Therefore, for application in a clinical workflow, preliminary analysis of the data subset may be necessary to identify the most reliable machine learning classifier before training of the entire cohort using a specific machine learning classifier.

As seen in Supplementary Table 1 and the aforementioned online link, the TCGA cohort consisted of heterogeneous imaging protocols from different MRI vendors with different field strengths, which may have contributed to the poor performance in the prediction of nonenhancing LGG grade. Apart from the heterogeneity of the MRI protocols, there are several possible explanations for the poor performance in the prediction of nonenhancing LGG grade in external validation in our study. First, although the absence of enhancement does not always indicate grade II glioma, enhancement is still an important indicator of a highergrade glioma, given that $52-85 \%$ of grade III gliomas show enhancement whereas only $47.9-56 \%$ of grade II gliomas show enhancement $(14,17,19,35)$. In the nonenhancing LGG subgroup, the radiomics classifiers were trained without this information on contrast enhancement. Moreover, the proportion of nonenhancing LGGs differed in the institutional and TCGA cohorts. The absence of crucial information regarding contrast enhancement as well as the discrepant proportion of nonenhancing LGG subgroups between cohorts may have resulted in the lower performance of the radiomics classifier. Second, in the external cohort, glioma grading was performed by various pathologists, which may have resulted in interobserver variability (36). One study has reported that $20-30 \%$ of gliomas are reclassified when the tumor material is independently reviewed (36). Histopathologic undergrading of LGGs may have also resulted in poor performance of the machine learning classifiers, especially in the heterogeneous TCGA cohort.

Our study has several limitations. First, it was based on a retrospectively collected dataset without an analysis of prognostic markers. Further studies are necessary to correlate grade and prognostic markers such as overall survival and progression-free survival with radiomics features. Second, although several studies have demonstrated the utility of incorporating advanced imaging techniques such as diffusion-weighted image or perfusion studies in radiomics $(24,37)$, such advanced imaging techniques were not included in our analyses. As these imaging techniques are routinely performed in tertiary centers, further studies including these advanced sequences should be performed. Third, the classifier performance in grading the nonenhancing LGG subgroup may have a limited reliability due to the small subgroup size with data 
imbalance between grade II and III tumors. Even though subsampling was performed to resolve the disparity in the frequencies of non-enhancement, there were variable performance levels according to the subsampling methods and machine learning algorithms, which may limit the reliability of the results. Further studies with a larger number of nonenhancing LGG cases are warranted for better assessment. Fourth, tumor segmentation was performed only at the hyperintense areas on FLAIR in our study. Segmentation for the contrast-enhancing portion of the tumor was not performed because the enhancing area was already included in the aforementioned ROI and its inclusion may lead to feature redundancy that may not be useful for the radiomics classifiers (38). Finally, interpolation was not used to create isotropic voxel spacing, and thus may not gain rotational invariability.

In conclusion, radiomics feature-based classifiers may be useful to predict the grade of LGGs. However, radiomics classifiers may have a limited value in the TCGA external validation cohort of the nonenhancing LGG subgroup.

\section{Supplementary Materials}

The Data Supplement is available with this article at https://doi.org/10.3348/kjr.2018.0814.

\section{Conflicts of Interest}

The authors have no potential conflicts of interest to disclose.

$$
\begin{aligned}
& \text { ORCID iDs } \\
& \text { Yoon Seong Choi } \\
& \text { https://orcid.org/0000-0002-6034-9912 } \\
& \text { Yae Won Park } \\
& \text { https://orcid.org/0000-0001-8907-5401 } \\
& \text { Sung Soo Ahn } \\
& \text { https://orcid.org/0000-0002-0503-5558 } \\
& \text { Jong Hee Chang } \\
& \text { https://orcid.org/0000-0003-1509-9800 } \\
& \text { Se Hoon Kim } \\
& \text { https://orcid.org/0000-0001-7516-7372 } \\
& \text { Seung-Koo Lee } \\
& \text { https://orcid.org/0000-0001-5646-4072 }
\end{aligned}
$$

\section{REFERENCES}

1. Cancer Genome Atlas Research Network, Brat DJ, Verhaak
RG, Aldape KD, Yung WK, Salama SR, Cooper LA, et al. Comprehensive, integrative genomic analysis of diffuse lowergrade gliomas. N Engl J Med 2015;372:2481-2498

2. Louis DN, Perry A, Reifenberger G, von Deimling A, FigarellaBranger D, Cavenee WK, et al. The 2016 World Health Organization classification of tumors of the central nervous system: a summary. Acta Neuropathol 2016;131:803-820

3. Rollin N, Guyotat J, Streichenberger N, Honnorat J, Tran Minh VA, Cotton F. Clinical relevance of diffusion and perfusion magnetic resonance imaging in assessing intra-axial brain tumors. Neuroradiology 2006;48:150-159

4. Lee EJ, Lee SK, Agid R, Bae JM, Keller A, Terbrugge K. Preoperative grading of presumptive low-grade astrocytomas on MR imaging: diagnostic value of minimum apparent diffusion coefficient. AJNR Am J Neuroradiol 2008;29:18721877

5. Olar A, Wani KM, Alfaro-Munoz KD, Heathcock LE, van Thuijl $\mathrm{HF}$, Gilbert MR, et al. IDH mutation status and role of WHO grade and mitotic index in overall survival in grade II-III diffuse gliomas. Acta Neuropathol 2015;129:585-596

6. Reuss DE, Mamatjan Y, Schrimpf D, Capper D, Hovestadt V, Kratz A, et al. IDH mutant diffuse and anaplastic astrocytomas have similar age at presentation and little difference in survival: a grading problem for WHO. Acta Neuropathol 2015;129:867-873

7. Killela PJ, Pirozzi CJ, Healy P, Reitman ZJ, Lipp E, Rasheed BA, et al. Mutations in IDH1, IDH2, and in the TERT promoter define clinically distinct subgroups of adult malignant gliomas. Oncotarget 2014;5:1515-1525

8. Wong JC, Provenzale JM, Petrella JR. Perfusion MR imaging of brain neoplasms. AJR Am J Roentgenol 2000;174:1147-1157

9. Cho SK, Na DG, Ryoo JW, Roh HG, Moon CH, Byun HS, et al. Perfusion MR imaging: clinical utility for the differential diagnosis of various brain tumors. Korean $\mathrm{J}$ Radiol 2002;3:171-179

10. Feiden W, Steude U, Bise K, Gündisch 0. Accuracy of stereotactic brain tumor biopsy: comparison of the histologic findings in biopsy cylinders and resected tumor tissue. Neurosurg Rev 1991;14:51-56

11. Yu X, Liu Z, Tian Z, Li S, Huang H, Xiu B, et al. Stereotactic biopsy for intracranial space-occupying lesions: clinical analysis of 550 cases. Stereotact Funct Neurosurg 2000;75:103-108

12. Field M, Witham TF, Flickinger JC, Kondziolka D, Lunsford LD. Comprehensive assessment of hemorrhage risks and outcomes after stereotactic brain biopsy. J Neurosurg 2001;94:545-551

13. Takano K, Kinoshita M, Arita H, Okita Y, Chiba Y, Kagawa N, et al. Diagnostic and prognostic value of 11C-methionine PET for nonenhancing gliomas. AJNR Am J Neuroradiol 2016;37:44-50

14. Tynninen 0 , Aronen $\mathrm{HJ}$, Ruhala M, Paetau A, Von Boguslawski $K$, Salonen 0 , et al. MRI enhancement and microvascular density in gliomas. Correlation with tumor cell proliferation. Invest Radiol 1999;34:427-434

15. Lüdemann L, Grieger W, Wurm R, Budzisch M, Hamm B, Zimmer 
C. Comparison of dynamic contrast-enhanced MRI with WHO tumor grading for gliomas. Eur Radiol 2001;11:1231-1241

16. Butler AR, Horii SC, Kricheff II, Shannon MB, Budzilovich GN. Computed tomography in astrocytomas. A statistical analysis of the parameters of malignancy and the positive contrastenhanced CT scan. Radiology 1978;129:433-439

17. White ML, Zhang Y, Kirby P, Ryken TC. Can tumor contrast enhancement be used as a criterion for differentiating tumor grades of oligodendrogliomas? AJNR Am J Neuroradiol 2005;26:784-790

18. Maia AC Jr, Malheiros SM, da Rocha AJ, da Silva CJ, Gabbai $A A$, Ferraz FA, et al. MR cerebral blood volume maps correlated with vascular endothelial growth factor expression and tumor grade in nonenhancing gliomas. AJNR Am J Neuroradiol 2005;26:777-783

19. Scott JN, Brasher PM, Sevick RJ, Rewcastle NB, Forsyth PA. How often are nonenhancing supratentorial gliomas malignant? A population study. Neurology 2002;59:947-949

20. Barker FG 2nd, Chang SM, Huhn SL, Davis RL, Gutin PH, McDermott MW, et al. Age and the risk of anaplasia in magnetic resonance-nonenhancing supratentorial cerebral tumors. Cancer 1997;80:936-941

21. Liu X, Tian W, Kolar B, Yeaney GA, Qiu X, Johnson MD, et al. MR diffusion tensor and perfusion-weighted imaging in preoperative grading of supratentorial nonenhancing gliomas. Neuro Oncol 2011;13:447-455

22. Davnall F, Yip CS, Ljungqvist G, Selmi M, Ng F, Sanghera B, et al. Assessment of tumor heterogeneity: an emerging imaging tool for clinical practice? Insights Imaging 2012;3:573-589

23. Zhou H, Vallières $M$, Bai HX, Su C, Tang H, Oldridge $D$, et al. MRI features predict survival and molecular markers in diffuse lower-grade gliomas. Neuro Oncol 2017;19:862-870

24. Kickingereder $P$, Burth S, Wick A, Götz M, Eidel 0, Schlemmer $\mathrm{HP}$, et al. Radiomic profiling of glioblastoma: identifying an imaging predictor of patient survival with improved performance over established clinical and radiologic risk models. Radiology 2016;280:880-889

25. Ceccarelli M, Barthel FP, Malta TM, Sabedot TS, Salama SR, Murray BA, et al. Molecular profiling reveals biologically discrete subsets and pathways of progression in diffuse glioma. Cell 2016;164:550-563

26. Bakas S, Akbari H, Sotiras A, Bilello M, Rozycki M, Kirby JS, et al. Advancing The Cancer Genome Atlas glioma MRI collections with expert segmentation labels and radiomic features. Sci Data 2017;4:170117

27. Shinohara RT, Sweeney EM, Goldsmith J, Shiee N, Mateen FJ,
Calabresi PA, et al.; Australian Imaging Biomarkers Lifestyle Flagship Study of Ageing; Alzheimer's Disease Neuroimaging Initiative. Statistical normalization techniques for magnetic resonance imaging. Neuroimage Clin 2014;6:9-19

28. van Griethuysen JJM, Fedorov A, Parmar C, Hosny A, Aucoin N, Narayan $V$, et al. Computational radiomics system to decode the radiographic phenotype. Cancer Res 2017;77:e104-e107

29. Chawla NV, Bowyer KW, Hall LO, Kegelmeyer WP. SMOTE: synthetic minority over-sampling technique. J Artif Intell Res 2002;16:321-357

30. Kuhn M. Building predictive models in $\mathrm{R}$ using the caret package. J Stat Softw 2008;28:1-26

31. Hilario A, Ramos A, Perez-Nuñez A, Salvador E, Millan JM, Lagares $A$, et al. The added value of apparent diffusion coefficient to cerebral blood volume in the preoperative grading of diffuse gliomas. AJNR Am J Neuroradiol 2012;33:701-707

32. Stadlbauer A, Gruber S, Nimsky C, Fahlbusch R, Hammen T, Buslei $\mathrm{R}$, et al. Preoperative grading of gliomas by using metabolite quantification with high-spatial-resolution proton MR spectroscopic imaging. Radiology 2006;238:958-969

33. Yu J, Shi Z, Lian Y, Li Z, Liu T, Gao Y, et al. Noninvasive IDH1 mutation estimation based on a quantitative radiomics approach for grade II glioma. Eur Radiol 2017;27:3509-3522

34. Park YW, Han K, Ahn SS, Choi YS, Chang JH, Kim SH, et al. Whole-tumor histogram and texture analyses of DTI for evaluation of IDH1-mutation and 1p/19q-codeletion status in World Health Organization grade II gliomas. AJNR Am J Neuroradiol 2018;39:693-698

35. Mihara F, Numaguchi $Y$, Rothman M, Kristt D, Fiandaca M, Swallow L. Non-enhancing supratentorial malignant astrocytomas: MR features and possible mechanisms. Radiat Med 1995;13:11-17

36. van den Bent MJ. Interobserver variation of the histopathological diagnosis in clinical trials on glioma: a clinician's perspective. Acta Neuropathol 2010;120:297-304

37. Kim JY, Park JE, Jo Y, Shim WH, Nam SJ, Kim JH, et al. Incorporating diffusion- and perfusion-weighted MRI into a radiomics model improves diagnostic performance for pseudoprogression in glioblastoma patients. Neuro Oncol 2019;21:404-414

38. Lee M, Woo B, Kuo MD, Jamshidi N, Kim JH. Quality of radiomic features in glioblastoma multiforme: impact of semiautomated tumor segmentation software. Korean J Radiol 2017;18:498-509 